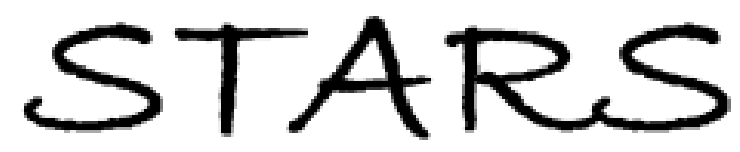

University of Central Florida

STARS

$1-1-2004$

\title{
Two-coefficient Cauchy model for low birefringence liquid crystals
}

Jun Li

University of Central Florida

Shin-Tson Wu

University of Central Florida

Find similar works at: https://stars.library.ucf.edu/facultybib2000 University of Central Florida Libraries http://library.ucf.edu

This Article is brought to you for free and open access by the Faculty Bibliography at STARS. It has been accepted for inclusion in Faculty Bibliography 2000 s by an authorized administrator of STARS. For more information, please contactSTARS@ucf.edu.

\section{Recommended Citation}

$\mathrm{Li}$, Jun and Wu, Shin-Tson, "Two-coefficient Cauchy model for low birefringence liquid crystals" (2004).

Faculty Bibliography 2000s. 4539.

https://stars.library.ucf.edu/facultybib2000/4539

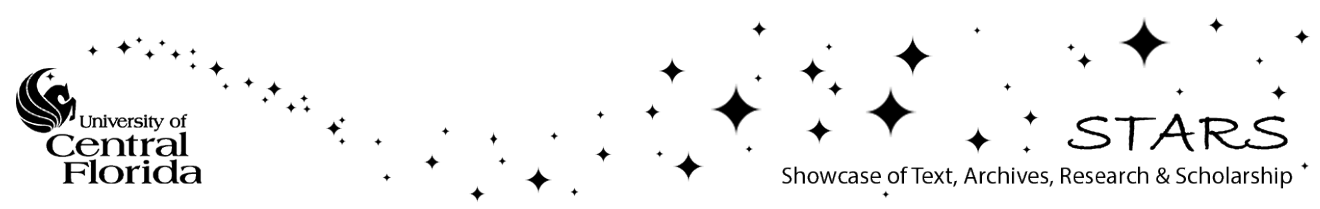




\section{Two-coefficient Cauchy model for low birefringence liquid crystals}

Cite as: Journal of Applied Physics 96, 170 (2004); https://doi.org/10.1063/1.1738526

Submitted: 14 January 2004 . Accepted: 16 March 2004 . Published Online: 17 June 2004

Jun Li, and Shin-Tson Wu

\section{ARTICLES YOU MAY BE INTERESTED IN}

Extended Cauchy equations for the refractive indices of liquid crystals

Journal of Applied Physics 95, 896 (2004); https://doi.org/10.1063/1.1635971

Temperature effect on liquid crystal refractive indices

Journal of Applied Physics 96, 19 (2004); https://doi.org/10.1063/1.1757034

Infrared refractive indices of liquid crystals

Journal of Applied Physics 97, 073501 (2005); https://doi.org/10.1063/1.1877815

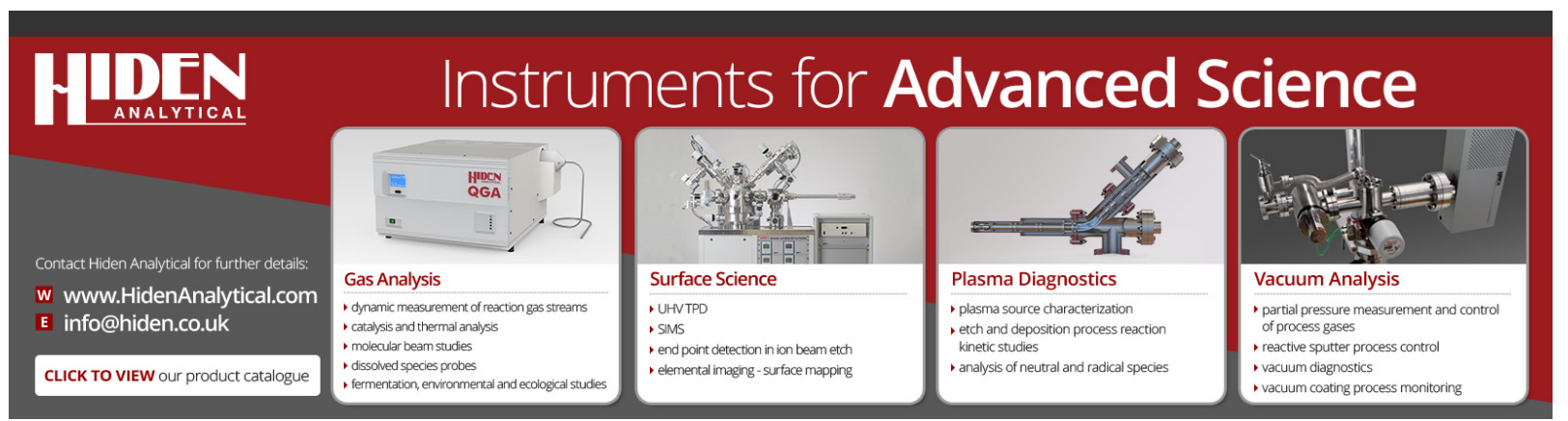




\title{
Two-coefficient Cauchy model for low birefringence liquid crystals
}

\author{
Jun Li and Shin-Tson Wua) \\ School of Optics/CREOL, University of Central Florida, Orlando, Florida 32816
}

(Received 14 January 2004; accepted 16 March 2004)

\begin{abstract}
The three- and two-coefficient Cauchy equations are derived based on the three-band model for the wavelength- and temperature-dependent refractive indices of anisotropic liquid crystals. For high birefringence $(\Delta n \geqslant 0.2)$ liquid crystals, the three-coefficient Cauchy model fits experimental results more accurately than the two-coefficient model. For low birefringence $(\Delta n \leqslant 0.12)$ liquid crystal mixtures the two-coefficient Cauchy model works equally well as the three-coefficient model in the off-resonance spectral region. (C) 2004 American Institute of Physics. [DOI: 10.1063/1.1738526]
\end{abstract}

\section{INTRODUCTION}

Thin-film-transistor liquid crystal displays (TFT-LCDs) have been commonly used in notebook and desktop computers, cellular phones, and projection displays. ${ }^{1,2}$ The fundamental light modulation mechanism of TFT-LCD is electric field induced liquid crystal (LC) refractive index change. The refractive indices of a LC are mainly determined by the molecular structure, wavelength, and temperature. To achieve a full-color display three primary colors (red, green, and blue) are needed. It is essential to know the wavelength- and temperature-dependent refractive indices of the LC mixture employed in order to optimize the cell design.

Several models have been developed to describe the wavelength and temperature dependencies of the LC refractive indices. ${ }^{3-8}$ Each model has its own merits and incom-

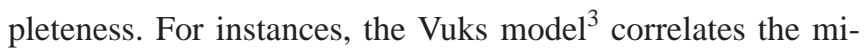
croscopic LC molecular polarizability to the macroscopic refractive indices. However, the wavelength and temperature effects are not described explicitly. The single band model $^{5,6}$ gives an explicit expression on the wavelength and temperature dependence for birefringence, but not for the individual refractive indices. The three-band model $^{7}$ describes the origins of the LC refractive indices for single LC compounds but requires three fitting parameters for each LC compound. If an LC mixture consists of a dozen different molecular structures, it would be too complicated for the three-band model to quantitatively describe the $\mathrm{LC}$ refractive indices of the mixture.

Although the original Cauchy equation ${ }^{9}$ was intended for the isotropic gases and liquids, it has been attempted to fit the wavelength-dependent refractive indices of some anisotropic liquid crystals. ${ }^{10,11}$ The fitting results are reasonably good except that the physical origins of the Cauchy coefficients are not clear. Recently, the extended Cauchy equations ${ }^{12}$ were derived for anisotropic liquid crystals based on the Vuks model. The extended Cauchy equations are applicable not only to single compounds but also to LC mixtures. Good fittings are found in the off-resonance region. However, there are three Cauchy coefficients involved for a) Author to whom correspondence should be addressed; electronic mail:
swu@mail.ucf.edu each refractive index. It is highly desirable to reduce the numbers of the fitting parameters.

In this article, we derive a two-coefficient Cauchy model for the refractive indices of low birefringence LC mixtures. We find that if the LC birefringence is below 0.12 the threecoefficient Cauchy equations can be reduced to two coefficients. In Sec. II, we show the derivation processes for the two-coefficient Cauchy equations. In Sec. III, the experimental method for measuring the refractive indices is briefly described. In Sec. IV, we validate the two-coefficient Cauchy equations by fitting the experimental data of two low birefringence TFT liquid crystal mixtures: one with positive dielectric anisotropy $(\Delta \epsilon)$ and the other with negative $\Delta \epsilon$. Excellent agreement between theory and experiment is obtained.

\section{THEORY}

In the three-band model, the refractive indices $\left(n_{e}\right.$ and $n_{o}$ ) are expressed as follows: ${ }^{7}$

$$
\begin{aligned}
& n_{e} \cong 1+g_{0 e} \frac{\lambda^{2} \lambda_{0}^{2}}{\lambda^{2}-\lambda_{0}^{2}}+g_{1 e} \frac{\lambda^{2} \lambda_{1}^{2}}{\lambda^{2}-\lambda_{1}^{2}}+g_{2 e} \frac{\lambda^{2} \lambda_{2}^{2}}{\lambda^{2}-\lambda_{2}^{2}}, \\
& n_{o} \cong 1+g_{0 o} \frac{\lambda^{2} \lambda_{0}^{2}}{\lambda^{2}-\lambda_{0}^{2}}+g_{1 o} \frac{\lambda^{2} \lambda_{1}^{2}}{\lambda^{2}-\lambda_{1}^{2}}+g_{2 o} \frac{\lambda^{2} \lambda_{2}^{2}}{\lambda^{2}-\lambda_{2}^{2}} .
\end{aligned}
$$

Here, $\lambda_{0}, \lambda_{1}$, and $\lambda_{2}$ (with $\lambda_{2}>\lambda_{1}$ ) denote the resonance wavelengths of the $\sigma \rightarrow \sigma^{*}$ and two $\pi \rightarrow \pi^{*}$ transitions, and $g_{0}, g_{1}$, and $g_{2}$ are the corresponding proportionality constants that depend on the oscillator strength and temperature. For a conjugated LC molecule, its $\lambda_{0}$ band is located in the vacuum ultraviolet region $\left(\lambda_{0} \sim 120 \mathrm{~nm}\right), \lambda_{1}$ is around 190 $210 \mathrm{~nm}$; not too sensitive to the LC structure, and $\lambda_{2}$ increases substantially as the molecular conjugation increases. For example, for the 4-cyano-4-n-pentyle-cyclohexanephenyl (5PCH) LC compound, its $\lambda_{1} \sim 200 \mathrm{~nm}$ and $\lambda_{2}$ $\sim 235 \mathrm{~nm}$ while for the 4-cyano-4-n-pentylbiphenyl (5CB) its $\lambda_{1}$ shifts to $210 \mathrm{~nm}$ and $\lambda_{2}$ shifts to $282 \mathrm{~nm}^{7}$

Equation (1) can be rewritten as follows: 


$$
\begin{aligned}
& n_{e} \cong 1+g_{0 e} \frac{\lambda_{0}^{2}}{1-\left(\frac{\lambda_{0}}{\lambda}\right)^{2}}+g_{1 e} \frac{\lambda_{1}^{2}}{1-\left(\frac{\lambda_{1}}{\lambda}\right)^{2}}+g_{2 e} \frac{\lambda_{2}^{2}}{1-\left(\frac{\lambda_{2}}{\lambda}\right)^{2}}, \\
& n_{o} \cong 1+g_{0 o} \frac{\lambda_{0}^{2}}{1-\left(\frac{\lambda_{0}}{\lambda}\right)^{2}}+g_{1 o} \frac{\lambda_{1}^{2}}{1-\left(\frac{\lambda_{1}}{\lambda}\right)^{2}}+g_{2 o} \frac{\lambda_{2}^{2}}{1-\left(\frac{\lambda_{2}}{\lambda}\right)^{2}} .
\end{aligned}
$$

In the off-resonance region, $\lambda>\lambda_{i}, i=0,1,2$, the terms $\lambda_{i}^{2} / \lambda^{2}(i=0,1,2)$ in the denominator are much smaller than 1 and Eq. (2) can be expanded into power series. Keeping the first three terms, we obtain

$$
\begin{aligned}
n_{e} \cong & 1+g_{0 e} \lambda_{0}^{2}\left(1+\frac{\lambda_{0}^{2}}{\lambda^{2}}+\frac{\lambda_{0}^{4}}{\lambda^{4}}\right)+g_{1 e} \lambda_{1}^{2}\left(1+\frac{\lambda_{1}^{2}}{\lambda^{2}}+\frac{\lambda_{1}^{4}}{\lambda^{4}}\right) \\
& +g_{2 e} \lambda_{2}^{2}\left(1+\frac{\lambda_{2}^{2}}{\lambda^{2}}+\frac{\lambda_{2}^{4}}{\lambda^{4}}\right), \\
n_{o} \cong & 1+g_{0 o} \lambda_{0}^{2}\left(1+\frac{\lambda_{0}^{2}}{\lambda^{2}}+\frac{\lambda_{0}^{4}}{\lambda^{4}}\right)+g_{1 o} \lambda_{1}^{2}\left(1+\frac{\lambda_{1}^{2}}{\lambda^{2}}+\frac{\lambda_{1}^{4}}{\lambda^{4}}\right) \\
& +g_{2 o} \lambda_{2}^{2}\left(1+\frac{\lambda_{2}^{2}}{\lambda^{2}}+\frac{\lambda_{2}^{4}}{\lambda^{4}}\right) .
\end{aligned}
$$

Grouping the similar terms together, we obtain the extended Cauchy equations

$$
\begin{aligned}
& n_{e} \cong A_{e}+\frac{B_{e}}{\lambda^{2}}+\frac{C_{e}}{\lambda^{4}}, \\
& n_{o} \cong A_{o}+\frac{B_{o}}{\lambda^{2}}+\frac{C_{o}}{\lambda^{4}},
\end{aligned}
$$

where

$$
\begin{aligned}
& A_{e}=1+g_{0 e} \lambda_{0}^{2}+g_{1 e} \lambda_{1}^{2}+g_{2 e} \lambda_{2}^{2}, \\
& B_{e}=g_{0 e} \lambda_{0}^{4}+g_{1 e} \lambda_{1}^{4}+g_{2 e} \lambda_{2}^{4}, \\
& C_{e}=g_{0 e} \lambda_{0}^{6}+g_{1 e} \lambda_{1}^{6}+g_{2 e} \lambda_{2}^{6},
\end{aligned}
$$

and

$$
\begin{aligned}
& A_{o}=1+g_{0 o} \lambda_{0}^{2}+g_{1 o} \lambda_{1}^{2}+g_{2 o} \lambda_{2}^{2}, \\
& B_{o}=g_{0 o} \lambda_{0}^{4}+g_{1 o} \lambda_{1}^{4}+g_{2 o} \lambda_{2}^{4}, \\
& C_{e}=g_{0 o} \lambda_{0}^{6}+g_{1 o} \lambda_{1}^{6}+g_{2 o} \lambda_{2}^{6} .
\end{aligned}
$$

Therefore, the three-coefficient Cauchy equations are derived from the three-band model and are applicable to the anisotropic media. Each Cauchy coefficient is related to the resonance wavelengths $\left(\lambda_{i}\right)$ and transition intensity $\left(g_{i}\right)$ as shown in Eqs. (5) and (6). For instance, if a LC compound contains only $\sigma$ electrons (e.g., cyclohexane rings), then $\lambda_{1}$ and $\lambda_{2}$ do not exist. The $n_{e}$ and $n_{o}$ are determined solely by the $\lambda_{\mathrm{o}}$ terms. As a result, the ABC coefficients would be small and the refractive indices in the visible region would be relatively small and insensitive to the wavelength. On the other hand, a linearly conjugated LC would exhibit a longer $\lambda_{2}$ and larger $g_{2}$ so that its refractive indices, especially birefringence $\left(\Delta n=n_{e}-n_{o}\right)$, would be greatly enhanced. From Eq. (4), both $n_{e}$ and $n_{o}$ decrease as the wavelength increases. In the long wavelength regime where $\lambda \gg \lambda_{2}, n_{e}$ and $n_{o}$ are reduced to $A_{e}$ and $A_{o}$, respectively, and are insensitive to the wavelength. Hence, birefringence $\left(\Delta n=A_{e}\right.$ $-A_{o}$ ) is also insensitive to the wavelength. This result is consistent with the prediction of the single band model. ${ }^{5}$

A LC mixture usually consists of several single compounds in order to widen the nematic range. Let us assume there are $m$ compounds in the LC mixture and each compound contributes a molar fraction $X_{i}(\mathrm{i}=1,2, \ldots . m)$ to the refractive indices of the mixture. The refractive indices $\left(n_{e i}\right.$ and $n_{o i}$ ) of the $i$ th component are expressed by Eq. (4). The refractive index of the mixture is a superposition of the individual components:

$$
\begin{aligned}
& n_{e} \cong \sum_{i=1}^{m} X_{i}\left(A_{e i}+\frac{B_{e i}}{\lambda^{2}}+\frac{C_{e i}}{\lambda^{4}}\right), \\
& n_{o} \cong \sum_{i=1}^{m} X_{i}\left(A_{o i}+\frac{B_{o i}}{\lambda^{2}}+\frac{C_{o i}}{\lambda^{4}}\right), \\
& X_{1}+X_{2}+\cdots+X_{m}=1 .
\end{aligned}
$$

Let $A_{e, o}^{\prime}=\sum_{i=1}^{m} X_{i}\left(A_{e, o}\right)_{i}, \quad B_{e, o}^{\prime}=\sum_{i=1}^{m} X_{i}\left(B_{e, o}\right)_{i}$, and $C_{e, o}^{\prime}$ $=\sum_{i=1}^{m} X_{i}\left(C_{e, o}\right)_{i}$, Eqs. (7a) and (7b) are reduced to

$$
\begin{aligned}
& n_{e} \cong A_{e}^{\prime}+\frac{B_{e}^{\prime}}{\lambda^{2}}+\frac{C_{e}^{\prime}}{\lambda^{4}}, \\
& n_{o} \cong A_{o}^{\prime}+\frac{B_{o}^{\prime}}{\lambda^{2}}+\frac{C_{o}^{\prime}}{\lambda^{4}} .
\end{aligned}
$$

Equation (8) represents the refractive indices of a LC mixture and has the same form as Eq. (4) which is for LC compounds, except for different Cauchy coefficients.

The three-coefficient Cauchy equations have been used to fit experimental results of LC mixtures. Good agreement is found in the off-resonance region. ${ }^{12}$ An undesirable feature is that it involves three fitting parameters. To reduce the fitting parameters to two, we need to prove that the third terms, i.e., the $\lambda^{-4}$ terms, in Eq. (8) can be ignored under certain conditions.

The fluorinated liquid crystals ${ }^{13,14}$ exhibit a high resistivity, low viscosity, and excellent material stability, and have become the mainstream for direct-view and projection displays. For a $90^{\circ}$ twisted nematic cell, to satisfy the GoochTarry first minimum condition ${ }^{15}$ the required $d \Delta n / \lambda$ is equal to $\sqrt{3} / 2$. A cell gap $d \sim 4-5 \mu \mathrm{m}$ is commonly chosen in order to achieve high manufacturing yield. For the green band centered at $\lambda=550 \mathrm{~nm}$, the LC birefringence should be in the vicinity of 0.1 . The fluorinated cyclohexane phenyl has $\Delta n$ in this range. The $\lambda_{2}$ band of such a LC structure occurs at $\sim 210 \mathrm{~nm}$ which is far from the visible (i.e., $\lambda_{2} \ll \lambda$ ) and, moreover, its transition oscillator strength is weak, ${ }^{16}$ which means the $g_{2 e, o}$ coefficients are small.

From Eqs. (4a) and (5c), the $C_{e} / \lambda^{4}$ term of the single LC compound can be rewritten as 


$$
\frac{C_{e}}{\lambda^{4}}=g_{0 e} \lambda_{0}^{2}\left(\frac{\lambda_{0}}{\lambda}\right)^{4}+g_{1 e} \lambda_{1}^{2}\left(\frac{\lambda_{1}}{\lambda}\right)^{4}+g_{2 e} \lambda_{2}^{2}\left(\frac{\lambda_{2}}{\lambda}\right)^{4} .
$$

For a low birefringence LC compound in the off-resonance region, all the $\left(\lambda_{i} / \lambda\right)^{4}$ terms (where $i=0,1$, and 2) in Eq. (9) are relatively small so that the $C_{e} / \lambda^{4}$ term can be ignored. Similarly, the third term for $n_{o}$ as shown in Eq. (4b) can be neglected, too. As a consequence, the refractive indices of a low birefringence LC compound are reduced to

$$
\begin{aligned}
& n_{e} \cong A_{e}+\frac{B_{e}}{\lambda^{2}}, \\
& n_{o} \cong A_{o}+\frac{B_{o}}{\lambda^{2}} .
\end{aligned}
$$

Based on the same arguments shown in Eq. (7), Eq. (10) holds equally well for low birefringence LC mixtures. From Eq. (10), there are only two Cauchy coefficients for each refractive index. If we measure the refractive index at two wavelengths, then the two Cauchy coefficients $(A$ and $B$ ) can be obtained and the refractive indices at any wavelength can then be calculated. The $A$ and $B$ coefficients are related to each band through Eqs. (5) and (6). Their physical meanings are clear.

\section{EXPERIMENT}

We measured the refractive indices of $5 \mathrm{CB}, 5 \mathrm{PCH}$, a commercial low birefringence fluorinated TFT LC mixture (Merck MLC-6241-000; $\Delta \epsilon=5.4$ ), and a fluorinated low birefringence negative $(\Delta \epsilon=-3)$ LC mixture (UCF-280) using a multiwavelength Abbe refractometer (Atago DR-M4) at $\lambda=450,486,546,589,633$, and $656 \mathrm{~nm}$. For some LC materials studied, their $n_{e}$ or $n_{o}$ is outside the measurement range at $\lambda=450$ and $486 \mathrm{~nm}$. Thus, we will have fewer experimental data. The accuracy of the Abbe refractometer is up to the fourth decimal. For a given wavelength, we measured the refractive indices of 5CB, 5PCH, MLC-6241-000, and UCF-280 from 10 to $55^{\circ} \mathrm{C}$. The temperature of the Abbe refractometer is controlled by a circulating constant temperature bath (Atago Model 60-C3).

To find the upper boundary of the two-coefficient Cauchy model [Eq. (10)], we compare its fitting results with the three-coefficient Cauchy model using the experimental data of 5CB, 5PCH, UCF-280, and MLC-6241-000. The data of $5 \mathrm{CB}$ and $5 \mathrm{PCH}$ are taken from Ref. 7 because these two compounds have the most complete experimental refractive

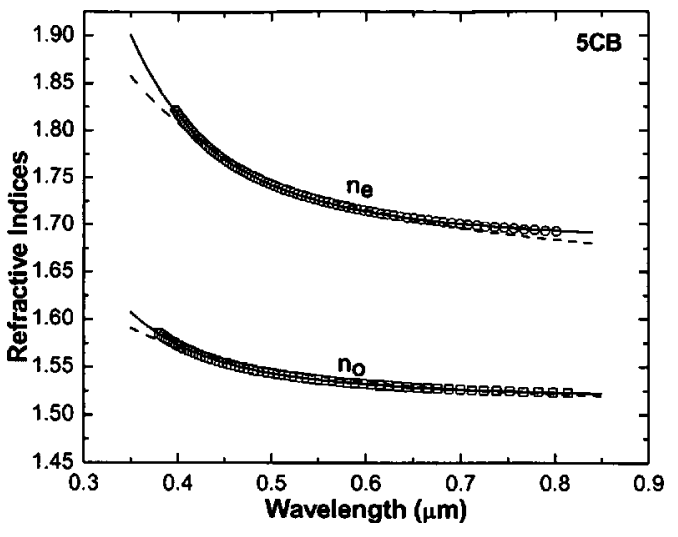

FIG. 1. Wavelength-dependent refractive index of $5 \mathrm{CB}$ at $T=25.1^{\circ} \mathrm{C}$. Open circles and squares are experimental data for $n_{e}$ and $n_{o}$, respectively. Solid lines are fittings using the three-coefficient Cauchy model [Eq. (4)] and dashed lines are fittings using the two-coefficient Cauchy model [Eq. (10)]. The fitting parameters are listed in Table I.

index data. At a given temperature, there are more than 50 refractive index data measured spanning in the $400-800 \mathrm{~nm}$ spectral range.

\section{RESULTS AND DISCUSSIONS}

\section{A. $5 \mathrm{CB}$}

Figure 1 depicts the wavelength-dependent refractive indices of $5 \mathrm{CB}$ at $T=25.1^{\circ} \mathrm{C}$. Dots are experimental data and solid lines are fitting results using the three-coefficient Cauchy model and dashed lines are for the two-coefficient Cauchy model. The fitting parameters for both models are listed in Table I. In the visible and near-infrared regions, the three-coefficient Cauchy model fits the experimental data very well. In Table I, the $\chi^{2}$ deviation is defined as the sum of the squares of observed values minus expected values and then divided by the expected values. For the three-coefficient Cauchy model, $\chi^{2}$ is small $\left(\sim 10^{-7}\right)$ indicating the fitting agrees with experimental results very well. On the other hand, the two-coefficient Cauchy model has a much larger $\chi^{2}$ deviation $\left(\sim 10^{-5}\right)$. This is because 5CB has a high birefringence $(\Delta n \sim 0.2)$ so that the contribution of the $\lambda^{-4}$ term to the refractive indices is still relatively large and cannot be neglected. From Table I, the $C / B$ ratio of the Cauchy coefficients is around $1 / 2$ for both $n_{e}$ and $n_{o}$. This indicates that the magnitude of the $\lambda^{-4}$ term is still comparable to the

\begin{tabular}{|c|c|c|c|c|c|c|c|c|}
\hline \multirow[b]{2}{*}{ Model } & \multicolumn{4}{|c|}{$n_{e}$} & \multicolumn{4}{|c|}{$n_{o}$} \\
\hline & $A_{e}$ & $B_{e}$ & $C_{e}$ & $\chi^{2}$ & $A_{o}$ & $B_{o}$ & $C_{o}$ & $\chi^{2}$ \\
\hline $\begin{array}{l}\text { Three-coefficient } \\
\text { Cauchy model }\end{array}$ & 1.6795 & 0.0048 & 0.0027 & $3.54 \times 10^{-7}$ & 1.5174 & 0.0022 & 0.0011 & $9.99 \times 10^{-8}$ \\
\hline $\begin{array}{l}\text { Two-coefficient } \\
\text { Cauchy model }\end{array}$ & 1.6427 & 0.0263 & & $2.00 \times 10^{-5}$ & 1.5152 & 0.0105 & & $7.47 \times 10^{-6}$ \\
\hline
\end{tabular}

TABLE I. Fitting parameters for the three- and two-coefficient Cauchy models: (LC) $5 \mathrm{CB}$ at $T=25.1{ }^{\circ} \mathrm{C}$. The units of Cauchy's $B$ and $C$ coefficients are $\mu \mathrm{m}^{2}$ and $\mu \mathrm{m}^{4}$, respectively. 


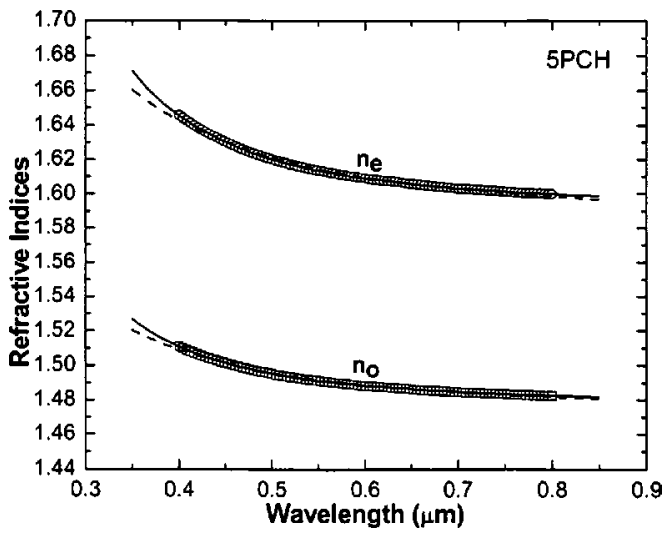

FIG. 2. Wavelength-dependent refractive indices of $5 \mathrm{PCH}$ at $T=25^{\circ} \mathrm{C}$. Open circles and squares are experimental data for $n_{e}$ and $n_{o}$, respectively. Solid lines are fittings using the three-coefficient Cauchy model [Eq. (4)] and dashed lines are fittings using the two-coefficient Cauchy model [Eq. (10)]. The fitting parameters are listed in Table II.

$\lambda^{-2}$ term and cannot be ignored. Therefore, for high birefringence $(\Delta n>0.2)$ LC compounds and mixtures the threecoefficient Cauchy model should be used.

\section{B. $5 \mathrm{PCH}$}

Figure 2 depicts the wavelength-dependent refractive indices of $5 \mathrm{PCH}$ at $T=25^{\circ} \mathrm{C}$. Dots are experimental data and solid lines are fitting results using the three-coefficient Cauchy model and dashed lines are for the two-coefficient Cauchy model. The fitting parameters for both models are listed in Table II. In the visible and near-infrared regions, the three-coefficient Cauchy model fits very well with the experimental data. The $\chi^{2}$ deviation is as small as $\sim 10^{-9}$. On the other hand, the two-coefficient Cauchy model also fits the data well. Although its $\chi^{2}$ deviation is still 2 orders of magnitude larger $\left(\sim 10^{-7}\right)$, both deviations are indistinguishable. This is because $5 \mathrm{PCH}$ has a relatively small birefringence $(\Delta n \sim 0.12)$ and the contribution of the $\lambda^{-4}$ term to the refractive indices is negligible. From Table II, the $C / B$ ratio of the Cauchy coefficients is $\sim 1 / 10$ for both $n_{e}$ and $n_{o}$. This indicates that the $\lambda^{-4}$ term is about one order of magnitude smaller than the $\lambda^{-2}$ term. Therefore, $\Delta \mathrm{n} \sim 0.12$ can be treated as the upper boundary that the two-coefficient Cauchy model begins to work as well as the three-coefficient Cauchy model.

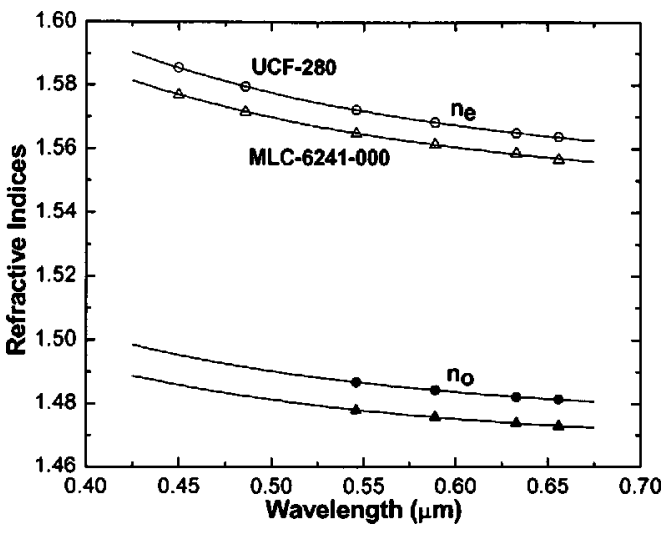

FIG. 3. Wavelength-dependent refractive indices of UCF-280 and MLC6241-000 at $T=25^{\circ} \mathrm{C}$. Open and filled circles are experimental data for $n_{e}$ and $n_{o}$ of UCF-280, respectively. Open and filled triangles are experimental data for $n_{e}$ and $n_{o}$ of MLC-6241-000, respectively. Solid lines are fittings by using the two-coefficient Cauchy model [Eq. (10)]. The fitting parameters are listed in Table III.

\section{Low birefringence LC mixtures}

To validate that the two-coefficient Cauchy model is applicable to the refractive indices of the low birefringence LC materials, we chose two LC mixtures for this study: Merck MLC-6241-000 $(\Delta \epsilon>0)$ and UCF-280 $(\Delta \epsilon<0)$. The negative $\Delta \epsilon$ LCs are particularly useful for homeotropic alignment, ${ }^{17}$ which exhibits an unprecedented contrast ratio. The refractive indices of these two mixtures were measured by our Abbe refractometer at $T=25^{\circ} \mathrm{C}$. Results are shown in Fig. 3.

In Fig. 3, circles and triangles represent the measured data for MLC-6241-000 and UCF-280, respectively, and solid lines are fitting results using the two-coefficient Cauchy model. The fitting parameters are listed in Table III. The $\chi^{2}$ deviation is as small as $\sim 10^{-8}$. We also fit the experimental data by using the three-coefficient Cauchy model, the two curves overlap almost exactly. From Fig. 3, the birefringence of MLC-6241-000 and UCF-280 is found to be around 0.085 at $\lambda=550 \mathrm{~nm}$. Therefore, for low birefringence LC compounds and mixtures, the two-coefficient Cauchy model works quite well.

\section{Temperature effect}

Figure 4 depicts the temperature-dependent birefringence of 5CB, 5PCH, UCF-280, and MLC-6241-000 measured at $\lambda=589 \mathrm{~nm}$. The squares, circles, open triangles, and

TABLE II. Fitting parameters for the three- and two-coefficient Cauchy models: (LC) $5 \mathrm{PCH}$ at $T=25^{\circ} \mathrm{C}$. The units of Cauchy's $B$ and $C$ coefficients are $\mu \mathrm{m}^{2}$ and $\mu \mathrm{m}^{4}$, respectively.

\begin{tabular}{|c|c|c|c|c|c|c|c|c|}
\hline \multirow[b]{2}{*}{ Model } & \multicolumn{4}{|c|}{$n_{e}$} & \multicolumn{4}{|c|}{$n_{o}$} \\
\hline & $A_{e}$ & $B_{e}$ & $C_{e}$ & $\chi^{2}$ & $A_{o}$ & $B_{o}$ & $C_{o}$ & $\chi^{2}$ \\
\hline $\begin{array}{l}\text { Three-coefficient } \\
\text { Cauchy model }\end{array}$ & 1.5903 & 0.0052 & 0.0006 & $5.13 \times 10^{-9}$ & 1.4763 & 0.0034 & 0.0003 & $1.59 \times 10^{-9}$ \\
\hline $\begin{array}{l}\text { Two-coefficient } \\
\text { Cauchy model }\end{array}$ & 1.5838 & 0.0094 & & $8.65 \times 10^{-7}$ & 1.4726 & 0.0058 & & $2.98 \times 10^{-7}$ \\
\hline
\end{tabular}


TABLE III. Fitting parameters for the two-coefficient Cauchy model: (LC) UCF-280 and MLC-6241-000 at $T=25^{\circ} \mathrm{C}$. The unit of Cauchy's $B$ coefficients is $\mu \mathrm{m}^{2}$.

\begin{tabular}{cccccccc}
\hline \hline \multirow{2}{*}{ Materials } & \multicolumn{3}{c}{$n_{e}$} & & \multicolumn{3}{c}{$n_{o}$} \\
\cline { 2 - 3 } \cline { 6 - 8 } \cline { 6 - 8 } & $A_{e}$ & $B_{e}$ & $\chi^{2}$ & & $A_{o}$ & $B_{o}$ & $\chi^{2}$ \\
\hline MLC-6241-000 & 1.5443 & 0.0083 & $1.51 \times 10^{-8}$ & & 1.4689 & 0.0053 & $1.20 \times 10^{-8}$ \\
& 1.5395 & 0.0076 & $4.68 \times 10^{-8}$ & & 1.4616 & 0.0049 & $9.69 \times 10^{-9}$ \\
\hline \hline
\end{tabular}

filled triangles are the measured birefringence of $5 \mathrm{CB}$, 5PCH, UCF-280, and MLC-6241-000, respectively, at different temperatures. The temperature range is from 10 to $55^{\circ} \mathrm{C}$. At room temperature and $\lambda=589 \mathrm{~nm}$, the birefringence of 5CB, 5PCH, UCF-280, and MLC-6241-000 is 0.2, 0.12, 0.0839 , and 0.0861 , respectively. The solid lines represent fittings using the Haller equation ${ }^{18}$

$$
\Delta n=(\Delta n)_{o}\left(1-T / T_{c}\right)^{\beta},
$$

where $(\Delta n)_{o}$ is the LC birefringence in the crystalline state, $\beta$ is a material constant, and $T_{c}$ is the clearing temperature of the LC material. The clearing point for $5 \mathrm{CB}, 5 \mathrm{PCH}, \mathrm{UCF}-$ 280, and MLC-6241-000 is 33.4, 52.9, 66.2, and $100^{\circ} \mathrm{C}$, respectively. From these fittings, we find $\left[(\Delta n)_{o}, \beta\right]$ $=[0.3505,0.1889],[0.1706,0.1512],[0.1426,0.2513]$, and $[0.1221,0.2209]$ for 5CB, 5PCH, UCF-280, and MLC-6241000, respectively. Although UCF-280 has a larger $(\Delta n)_{o}$ than MLC-6241-000, its clearing temperature is much lower. As a result, its birefringence at room temperature is lower than that of MLC-6241-000 due to the order parameter effect.

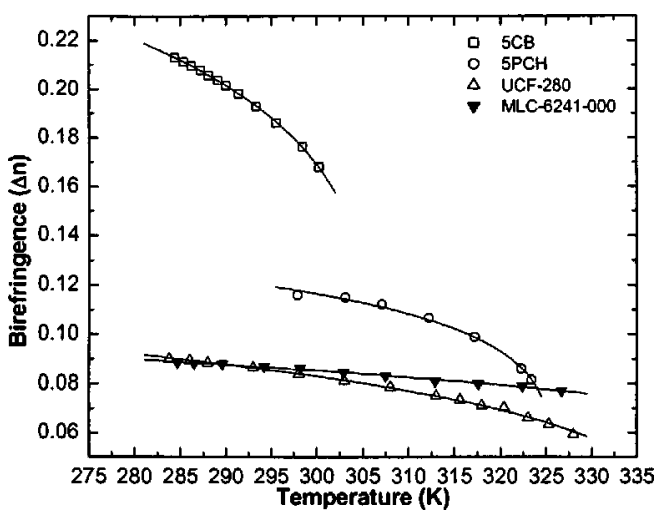

FIG. 4. Temperature-dependent birefringence $(\Delta n)$ of $5 \mathrm{CB}$ (open squares), 5PCH (open circles), UCF-280 (open upward-triangles), and MLC-6241000 (filled downward-triangles) at $\lambda=589 \mathrm{~nm}$. The four solid lines are fitting curves using $\Delta n=(\Delta n)_{o}\left(1-T / T_{c}\right)^{\beta}$, where $T_{c}$ is clearing point. For 5CB: $(\Delta n)_{o}=0.3505$ and $\beta=0.1889$. For 5PCH: $(\Delta n)_{o}=0.1706$ and $\beta=0.1512$. For UCF-280: $(\Delta n)_{o}=0.1426$ and $\beta=0.2513$. For MLC-6241-000: $(\Delta n)_{o}$ $=0.1221$ and $\beta=0.2209$.

\section{CONCLUSIONS}

We have derived and compared the three- and twocoefficient Cauchy models for describing the wavelengthand temperature-dependent refractive indices of LC compounds and mixtures based on the three-band model. If the LC birefringence is larger than 0.2, the three-coefficient Cauchy model has to be used. On the other hand, if the LC birefringence is smaller than $\sim 0.12$, the $\lambda^{-4}$ term can be ignored and the two-coefficient Cauchy model works equally well as the three-coefficient Cauchy model. Most of TFT LC mixtures developed for direct-view and projection displays have a relatively low birefringence. Thus, the two-coefficient Cauchy model is adequate.

\section{ACKNOWLEDGMENTS}

The authors are indebted to Dr. S. Gauza and Dr. X. Liang for technical assistance. This work is supported by AFOSR under Contract No. F49620-01-1-0377.

${ }^{1}$ E. H. Stupp and M. S. Brennesholtz, Projection Displays (Wiley, New York, 1998)

${ }^{2}$ S. T. Wu and D. K. Yang, Reflective Liquid Crystal Displays (Wiley, New York, 2001).

${ }^{3}$ M. F. Vuks, Opt. Spektrosk. 20, 644 (1966).

${ }^{4}$ W. H. de Jeu, Physical Properties of Liquid Crystalline Materials (Gordon and Breach, New York, 1980), Chap. 4.

${ }^{5}$ S. T. Wu, Phys. Rev. A 33, 1270 (1986).

${ }^{6}$ S. T. Wu, J. Appl. Phys. 69, 2080 (1991).

${ }^{7}$ S. T. Wu, C. S. Wu, M. Warenghem, and M. Ismaili, Opt. Eng. 32, 1775 (1993)

${ }^{8}$ E. M. Averyanov, J. Opt. Technol. 64, 417 (1997).

${ }^{9}$ M. Born and E. Wolf, Principle of Optics, 6th ed. (Pergamon, New York, 1980).

${ }^{10}$ H. Mada and S. Kobayashi, Mol. Cryst. Liq. Cryst. 33, 47 (1976).

${ }^{11}$ L. Pohl and U. Finkenzeller, Liquid Crystals: Applications and Uses, edited by B. Bahadur (World Scientific, Singapore, 1990), Vol. 1, Chap. 4.

${ }^{12}$ J. Li and S. T. Wu, J. Appl. Phys. 95, 896 (2004).

${ }^{13}$ G. Weber, U. Finkenzeller, T. Geelhaar, H. J. Plach, B. Rieger, and L. Pohl, Liq. Cryst. 5, 1381 (1989).

${ }^{14}$ R. Tarao, H. Saito, S. Sawada, and Y. Goto, SID Tech. Digest 25, 233 (1994)

${ }^{15}$ C. H. Gooch and H. A. Tarry, J. Phys. D 8, 1575 (1975).

${ }^{16}$ I. C. Khoo and S. T. Wu, Optics and Nonlinear Optics of Liquid Crystals (World Scientific, Singapore, 1993), Chap. 1.

${ }^{17}$ M. F. Schiekel and K. Fahrenschon, Appl. Phys. Lett. 19, 391 (1971).

${ }^{18}$ I. Haller, Prog. Solid State Chem. 10, 103 (1975). 